\title{
COMBINATION OF SEM AND LIGHT SCATTERING DATA FOR THE INVERSE ESTIMATION OF PARTICLE SIZE DISTRIBUTION USING A BAYESIAN APPROACH
}

\author{
G. L. Frontini ${ }^{1,2}$, F. A. Otero ${ }^{1,2}$, G. E. Eliçabe ${ }^{2}$ \\ ${ }^{1}$ Department of Mathematics, College of Engineering at the National University of Mar del \\ Plata (foterovega@fi.mdp.edu.ar) \\ ${ }^{2}$ Institute of Material Science and Technology, National Research Council and National Uni- \\ versity of Mar del Plata
}

\begin{abstract}
In this work, Static Light Scattering (SLS) measurements are used to estimate the Particle Size Distribution (PSD) of a polymeric particle system incorporating prior information obtained from measurements of Scanning Electron Microscopy (SEM). The inverse problem is solved using a Bayesian approach following two different schemes for the representation of the PSD. In the first one, the PSD is represented by a parameterized family of distributions in a fixed-form scheme. In the second one, there is no assumption on the shape of the PSD, i.e. a free-form scheme is used. The Metropolis-Hastings algorithm is used to solve the inverse problem. The proposed objective is to obtain results from experimental data that are more consistent with respect to those obtained with SEM in a previous work, providing trust confidence intervals for the solution.

The application of the Bayesian approach allows one to use, simultaneously, information provided by two different experimental techniques. The main conclusion of this work is that the usage of a Bayesian approach is recommended for cases where only an approximate model is available and use of reliable additional information can be made.
\end{abstract}

Keywords: Inverse problem, Bayesian estimation, Particle Size Distribution, Light scattering.

\section{INTRODUCTION}

Systems of polymeric particles suspended in a fluid appear in diverse industrial applications. These systems are usually described by their Particle Size Distribution (PSD). There are many experimental techniques for estimating the PSD. The optical ones stand out because they are non-destructive. One of the most important optical techniques is the so-called Static Light Scattering (SLS) which has been widely used in practice [1,2]. The basic process consists on illuminating a sample of particles by a laser light and measuring the intensity of the light scattered at different angles. The estimation of the PSD from SLS data requires solving an ill-conditioned inverse problem which gives shortly accurate results in some cases. In order 
to properly solve this type of inverse problems some kind of regularization is needed and several methods mostly based on a least-squares approach has been proposed [3, 4, 5]. However when noisy data are available and/or modeling inexactitude cannot be neglected, results obtained by this type of methods may be poorly consistent or even unreasonable. In these cases, using prior information on a Bayesian approach is an interesting alternative to get more reliable results.

In the next section, there is an introduction describing the used models for the calculation of the intensity of scattered light by a given particle system. This is the so-called direct problem. Then, the Bayesian approach is presented and studied in the section . At this point, the analysis is divided into two schemes according to the representation of the PSD: a fixedform scheme when the PSD is assumed to be properly expressed as a log-normal distribution, and a free-form scheme. This entire section describes the so-called inverse problem. The following section includes all remarkable aspects of the implementation. Later, a description of studied examples is provided and obtained results are discussed in the final section.

\section{DIRECT PROBLEM}

Appropriate use of models depends on both experimental setups and characteristics of analyzed systems. In this work, concentrated particle systems are studied under the RayleighDebye-Gans (RDG) regime which considers close values between refractive indexes of particles and surrounding medium (low contrast condition). Under these assumptions multiple scattering effects can be neglected and only interference between particles should be considered. Several models of different mathematical complexity were developed. In particular, this paper is interested in the Hard-Sphere (HS) models which represent the particles as impenetrable spheres which cannot be overlapped in space. One of these HS models under the Percus-Yevick (PY) approximation corresponds to the Finite Mixture Model (FMM) developed by Vrij $[6,7]$ which is rigorous under this approximation. A summarized expression for the FMM can be found in [8]. However, the FMM has also a strongly non-linear dependence on the PSD so its practical use in inverse problems has several problems including high error propagation and a very time-consuming computational implementation. In order to overcome these difficulties some approximations were built from the FMM, including the Local Monodisperse Approximation (LMA) introduced in [9]. The LMA supposes a spatial distribution of the particles according to their respective sizes and it expresses the scattered light intensity $I_{s}$ as the integral in eq.(1)

$$
I_{s}=K \int_{0}^{\infty} f(R) S\left(p_{H S}, q, R\right) F^{2}(q, R) d R
$$

where $f(R)$ represents the PSD expressed in terms of the radius $R . S\left(p_{H S}, q, R\right)$ is the socalled structure factor defined in eqs.(2) to (7), $p_{H S}$ is an effective particle density and $q$, expressed in eq.(8), is the modulus of the scattering vector $\mathbf{q} ; F^{2}(q, R)$ is the form factor defined in eq.(9) and $K$ is a global constant. 


$$
S\left(p_{H S}, q, R\right)=\frac{1}{N_{p}(2 \pi)^{3} C(q)}
$$

where

$$
\begin{aligned}
& N_{p}(2 \pi)^{3} C(q)= \\
& =24 p\left\{\frac{(\alpha+\beta+\delta)}{u^{2}} \cos u-\frac{(\alpha+2 \beta+4 \delta)}{u^{3}} \sin u-\right. \\
& \left.-\frac{2(\beta+6 \delta)}{u^{4}} \cos u+\frac{2 \beta}{u^{4}}+\frac{24 \delta}{u^{5}} \sin u+\frac{24 \delta}{u^{6}}(\cos u-1)\right\}
\end{aligned}
$$

and

$$
\begin{gathered}
\alpha=\frac{\left(1+2 p_{H S}\right)^{2}}{\left(1-p_{H S}\right)^{4}} \\
\beta=-6 p_{H S} \frac{\left(1+\frac{p_{H S}}{2}\right)^{2}}{\left(1-p_{H S}\right)^{4}} \\
\delta=\frac{p_{H S}\left(1+2 p_{H S}\right)^{2}}{2\left(1-p_{H S}\right)^{4}} \\
u=2 q R . \\
q=\frac{4 \pi n_{s}}{\lambda} \sin (\theta / 2) . \\
F(q, R)=\frac{1}{q} \int_{0}^{R} r \sin q r d r=\frac{1}{q^{3}}[\sin (q R)-q R \cos (q R)] .
\end{gathered}
$$

\section{INVERSE PROBLEM}

The main feature of the Bayesian approach is that every variable or parameter involved in the model is considered as a random variable so they must be described through their probability density function (pdf). Then, using the Bayes theorem, it is possible to obtain the socalled posterior pdf of the model parameters as in eq. (10):

$$
\pi_{\text {posterior }}(P)=\pi\left(P / I_{\varepsilon}\right)=\frac{\pi_{\text {prior }}(P) \pi\left(I_{\varepsilon} / P\right)}{\int_{P} \pi_{\text {prior }}(P) \pi\left(I_{\varepsilon} / P\right) d P} .
$$


where $\pi_{\text {posterior }}(P)$ is the posterior pdf of the parameters $P$ of the model, equivalent to the conditional pdf of the parameters $P$ given the experimental intensity measurements $I_{\varepsilon}$; $\pi_{\text {prior }}(P)$ is the prior pdf of the parameters $P$ and $\pi\left(I_{\varepsilon} / P\right)$ is the conditional pdf of measurements $I_{\varepsilon}$ given the parameters $P$, also called the likelihood function. The integral in eq.(10) is the pdf of measurements $\pi\left(I_{\varepsilon}\right)$ and it corresponds to a normalization constant.

In a statistical sense, the Bayesian solution is the set of parameters $P$ that maximizes $\pi_{\text {posterior }}(P)$, which is called the Maximum A Posteriori (MAP) solution, which implies that there is no need to compute $\pi\left(I_{\varepsilon}\right)$. However, the estimation of corresponding confidence intervals requires solving Bayes theorem. Depending on the number of parameters $P$, numerical computation of the integral can be a very time-consuming task. In order to reduce these computational costs alternative methods have been proposed, especially Monte Carlo Markov Chain (MCMC) techniques [10]. Most used MCMC techniques include Gibbs Sampling (GS) [11] and Metropolis-Hastings (MH) algorithm $[12,13]$. In this work the $\mathrm{MH}$ algorithm has been chosen because it has shown its versatility in many applications. A detailed analysis of the $\mathrm{MH}$ algorithm can be found in [14].

The goal of the $\mathrm{MH}$ algorithm is to build an adequate Markov chain $\mathbf{X}$ which simulates a distribution that has a density $\pi(P)$, defining a density $q_{T}\left(P^{(t+1)} / P^{(t)}\right)$, called candidate generating density, between a state of the Markov chain at a time $t$ (i.e. a value of the set of parameters $P^{(t)}$ ) and the next one $P^{(t+1)}$. Then, the $\mathrm{MH}$ algorithm is defined by two steps: a first step in which a proposed value is drawn from the candidate generating density and a second step in which the proposed value $P^{*}$ is accepted as the next iterate in the Markov chain according to the probability $\alpha_{M H}\left(P^{*} / P^{(t)}\right)$ defined as:

$$
\alpha_{M H}\left(P^{*} / P^{(t)}\right)=\min \left(\frac{\pi\left(P^{*} / I_{\varepsilon}\right) \cdot q_{T}\left(P^{*} / P^{(t)}\right)}{\pi\left(P^{(t)} / I_{\varepsilon}\right) \cdot q_{T}\left(P^{(t)} / P^{*}\right)}, 1\right) .
$$

If $\alpha_{M H}\left(P^{*} / P^{(t)}\right)>u$, where $u$ is a random variable of uniform distribution U[0,1], then the drawn sample $P^{(t+1)}$ is accepted and $P^{*}=P^{(t+1)}$; if $\alpha_{M H}\left(P^{*} / P^{(t)}\right) \leq u P^{(t+1)}$ is rejected and $P^{*}=P^{(t)}$. An important aspect of the algorithm is that the acceptance probability given by $\alpha_{M H}\left(P^{*} / P^{(t)}\right)$ avoids the computation of $\pi\left(I_{\varepsilon}\right)$.

Although the $\mathrm{MH}$ algorithm has a relatively simple implementation, it needs the fitting of several own parameters for the correct performance. In the same way, even when Bayes theorem is the keystone of the Bayesian approach, explicit forms of eq.(10) and its specific application differ in every developed scheme. These schemes are explained in what follows.

\subsection{Fixed-Form Scheme}

In this problem, the PSD can, sometimes, be represented by a log-normal distribution of parameters $g$ and $R_{0}$ as shown in eq.(12):

$$
f(R)=\frac{(g / \pi)^{1 / 2}}{R} \exp \left\{-g\left[\log \left(\frac{R}{R_{0}}\right)\right]^{2}\right\}
$$


Then, the set of parameters $P$ used in eq.(10) is defined as $P=\left[R_{0}, g, p_{H S}, K\right]^{T}$. Some sort of regularization of this inverse problem may be simply achieved by parameterizing the PSD. All previous information on these parameters expressed in a statistical manner is used in $\pi_{\text {prior }}(P)$. When there is no previous information on some parameters, it is said that the pdf of these parameters is non-informative and it is equal to a constant. A great simplification occurs when a subset of parameters $P^{*}$ with prior information can be modelled as a normal pdf. In this case, this pdf is expressed as:

$$
\pi_{\text {prior }}\left(P^{*}\right)=\frac{1}{(\sqrt{2 \pi}|\Sigma|)^{N^{*}}} \exp \left[-\frac{1}{2}\left(P^{*}-\overline{P^{*}}\right)^{T} \Sigma^{-1}\left(P^{*}-\overline{P^{*}}\right)\right]
$$

where $|\Sigma|$ is the determinant of the covariance matrix $\Sigma, N^{*}$ is the number of parameters in $P^{*}$ and $\overline{P^{*}}$ is the mean vector in this prior pdf.

In this work, prior information is obtained by Scanning Electron Microscopy (SEM). SEM micrographs bringing information on mean value $\bar{R}$ and variance $\sigma$ of the PSD and indirectly about parameters $g$ and $R_{0}$ using eqs.(14) and (15), which are used in eq.(13). Although SEM allows estimation of volume fraction of particles $\eta$, limitations and distortions in that estimation are important and the reliability in the results depends on the type of measured material [15]

$$
\begin{gathered}
\bar{R}=R_{0} \exp \left(\frac{1}{4 g}\right) \\
\sigma^{2}=2 R_{0}^{2} \exp \left(\frac{3}{4 g}\right) \operatorname{senh}\left(\frac{1}{4 g}\right)
\end{gathered}
$$

A similar explicit form for the likelihood function $\pi\left(I_{\varepsilon} / P\right)$ can be found when measurements can be modeled with a normal distribution. In this case,

$$
\pi\left(I_{\varepsilon} / P\right)=\frac{1}{(\sqrt{2 \pi})^{M}} \exp \left\{\left[I_{\varepsilon}-I_{s}(P)\right]^{T} W\left[I_{\varepsilon}-I_{s}(P)\right]\right\}
$$

where $M$ is the number of measurements, $W$ is the inverse of the covariance matrix of the measurements and $I_{s}$ are intensities generated by the LMA defined in eq.(1).

\subsection{Free-Form Scheme}

It is important to notice that the resulting problem displayed in eq.(1) has a linear dependence on $f(R)$ for a specified value for the parameter $p_{H S}$. In this case, when no assumption on the shape of $f(R)$ is considered, an explicit regularization is needed. This regularization is carried out on the prior pdf of eq.(10) where Bayes theorem results 


$$
\pi_{\text {posterior }}\left(f / I_{\varepsilon}, p_{H S}\right)=\frac{\pi\left(I_{\varepsilon} / f, p_{H S}\right) \pi_{\text {prior }}\left(f / p_{H S}\right)}{\int_{f} \pi\left(I_{\varepsilon} / f, p_{H S}\right) \pi_{\text {prior }}\left(f / p_{H S}\right) d f},
$$

where $\pi_{\text {posterior }}\left(f / I_{\varepsilon}, p_{H S}\right)$ is the conditional pdf for the PSD given the measurements and the parameter $p_{H S}, \pi_{p r i o r}\left(f / p_{H S}\right)$ is the prior pdf for the PSD given the parameter $p_{H S}$, $\pi\left(I_{\varepsilon} / f, p_{H S}\right)$ is the likelihood function with a similar expression to eq.(16) and the integral is once again a normalizing factor. In particular it is possible to include regularization, and SEM information on the prior pdf,

$$
\pi_{\text {prior }}\left(f / p_{H S}\right)=\pi_{\text {prior }}\left(P^{*}\right) \frac{1}{(\sqrt{2 \pi})^{N}} \exp \left\{-\left[\frac{1}{2} \gamma f^{T} H^{T} H f\right]\right\},
$$

where $N$ is the number of components of the discretized PSD, $H$ is a regularization matrix, $\gamma$ is an adjustable regularization parameter and $\pi_{\text {prior }}\left(P^{*}\right)$ is the prior information from SEM that has the form of eq.(13) where $P^{*}=[\bar{R}, \sigma]^{T}$

Finally, an Iterative Bayesian Method (IBM) using Bayes theorem in the form of eq.(17) was proposed. It can be described in the following steps:

1. Choose a value for parameter $p_{H S}$.

2. Compute a value for $\gamma$ using some method such as Generalized Cross Validation (GCV), L-Curve (LC) or Principle of Discrepancy (PD)

3. Estimate the PSD, $f(R)$, applying $\mathrm{MH}$ algorithm in the form of eq.(17)

4. Repeat the first three steps for the whole range of possible values of $p_{H S}$

5. Select the PSD with the maximum product $\pi\left(I_{\varepsilon} / f, p_{H S}\right) . \pi_{p r i o r}\left(f / p_{H S}\right)$

\section{IMPLEMENTATION}

Both schemes of the Bayesian approach were implemented on Matlab®. As mentioned in last section, a correct performance of the $\mathrm{MH}$ algorithm requires a good fitting of its own parameters. Such parameters include initial sample, prior information, acceptance ratio, candidate generating density, length of the Markov chain and samples considered in this chain. Convergence tests have been performed for different initial samples in order to determine if differences between obtained chains are significant. In this sense, while the fixed-form estimation had no convergence problems, the free-form scheme showed a high sensitivity to the initial sample. Actually, the $\mathrm{MH}$ algorithm has no warranty on solving multiple local minima problems. An alternative solution is to slightly modify the algorithm by including concepts from the Simulated Annealing (SA) algorithm [16]. The idea of the variant applied in this work is to create a distribution which exaggerates the probability value of the MAP solution, by changing the probability of $\alpha_{M H}\left(P^{*} / P^{(t)}\right)$ in eq.(11): 


$$
\alpha_{M H}\left(P^{*} / P^{(t)}\right)=\min \left(\left[\frac{\pi\left(P^{*} / I_{\varepsilon}\right)}{\pi\left(P^{(t)} / I_{\varepsilon}\right)}\right]^{t} \frac{q_{T}\left(P^{*} / P^{(t)}\right)}{q_{T}\left(P^{(t)} / P^{*}\right)}, 1\right) .
$$

Then, the idea is to reach this MAP solution and use it as an initial sample with the MH algorithm in its original form. Prior information is a critical factor in determining the posterior pdf. When the state of knowledge is primarily subjective, it is recommended to propose several priors and observe their effects on results. In this work, however prior information is well defined and based on alternative SEM experiments. In this context, a few priors from SEM measurements were simulated in a Monte Carlo routine that will be explained in next section. Acceptance ratio is the percentage of times a new sample is accepted; so, if a too small value is selected, then the chain slowly covers (or even does not cover) the whole distribution. However, choosing a too large value produces jumps very often. Best results showed that acceptance ratio should be set between 0.23 and 0.33 , in agreement with previous works [17]. It also must be specified a candidate generating density, typically from a family of distributions with tuning parameters such as scale and location. Here the so-called random walk was proposed. According to this method, a random variable $\varepsilon$ with distribution $q_{T}$ is added to the present state to generate next candidate. This parameter updating process is performed at one parameter at a time randomly selected. Finally, the length of the generated chain must be large enough to be a good approximation to the final distribution and first samples until reach stationary regime should be discarded. Final chains of 200000 samples and 300000 samples were respectively built for the fixed-form and free-form approaches. Other implementation issues were needed in the free-form approach. These include discretization of the PSD, selection of the regularization matrix $H$ and a method for computing the regularization parameter. The random walk where each candidate is generated changing one parameter at a time has a very slow performance and convergence problems if there is a huge number of components of the PSD. It was considered a maximum number of 40 points in the PSD to make the problem tractable. A few regularization matrices were studied. Best results in simulations were obtained for a matrix corresponding to a second derivative order. The computation of the regularization parameter was an important point to analyze since it has a great influence in the computed solution. In this work three methods were analyzed: GCV, LC and PD. It has been shown that GCV usually produces data overfitting [18] and hence it may have a bad performance on systems were modeling errors are important. LC, on the other hand, produces an over-regularization of the solution losing a good part of information provided by the data. Finally PD seemed to bring an intermediate solution for simulated cases and it was then used in the experimental examples. This method however requires an estimation of the noise level. 


\section{EXAMPLES}

\subsection{Simulated Examples}

Simulated examples were divided into two groups. In the first, measurements were generated adding numerical noise to scattered intensity $I_{s}$ obtained from the LMA. The noise follows a normal distribution with zero mean and standard deviation $\sigma_{\varepsilon}$ of $0.1 \%$ and $1 \%$ of the peak. In the second group, in an attempt to make measurements more realistic, measurements $I_{s}$ were simulated using the FMM. The simulated particle system is described by a lognormal distribution with parameters $g=10$ and $R_{0}=0.25$ (in a micrometer scale) and two volume fractions corresponding to different levels of concentration $\eta_{1}=0.001$ and $\eta_{2}=0.0216$. SEM estimations were also simulated, using a Monte Carlo routine. The developed process consists of a few steps. First the total population of particles is generated and located over a three-dimensional space. A sample of a certain number of particles (typically between several tens to few hundreds) is then taken over random positions in space. This sampling process is repeated until getting an acceptable number of samples to statistically analyze the ensemble. Final results are mean and variance of mean radius $\bar{R}$ and standard deviation $\sigma$ of the PSD (or alternatively $R_{0}$ and $g$ ).

\subsection{Experimental Examples}

Experimental examples were taken from previously reported publications $[19,20]$. In those works blends of polyisobutilene (PIB) labeled PIB5 and PIB025 in Isobornyl Methacrylate (IBoMA) were used. The blends contained 50\% of PIB025 (50PIB025 and 50PIB025_replica) and 30\% and 50\% of PIB5 (30PIB5 and 50PIB5). Refractive indices were 1.51 for PIB5 and PIB025 and 1.48 for IBoMA. SLS measurements were performed using a Flat Cell Light Scattering (FCLS) apparatus which consists of a linear array of photodiodes that detects the light scattered by a thin sample illuminated by a $17 \mathrm{~mW}$ He-Ne laser with random polarization. SEM micrographs were obtained using a Jeol JSM 6460 LV device. From these micrographs, estimations of mean values corresponding to mean radius $\bar{R}$ and standard deviation $\sigma$ of the PSD were obtained. Their respective variances were computed from the sample-error.

\section{RESULTS}

For diluted systems the LMA is an excellent approximation to the FMM, so modeling errors are not important and results obtained in simulated examples under these conditions (the particle system corresponding to $\eta_{1}=0.001$ ) are practically the same using both models to generate measurements. In this case, using a Bayesian approach with SLS measurements achieves a great performance and it can improve previous confidence intervals obtained with SEM simulations as much as an equivalent of a micrograph of over 40000 particles for noise of $\sigma_{\varepsilon}=0.1 \% \max \left(I_{\varepsilon}\right)$ and another of about 8000 particles for $\sigma_{\varepsilon}=1 \% \max \left(I_{\varepsilon}\right)$ 
For concentrated systems, measurements obtained with the LMA model can be quite different from those generated with the FMM. In this case, these differences produce distortions in estimations with the LMA model when using FMM data. In this sense, when modeling errors cannot be neglected, a modification of the estimated measurement error represented in the elements of $W$ in eq.(16) was proposed by adding another term to the normal noise. This term corresponds to an error represented by a gaussian process, interpolated over each value of measured $q$, between intensities generated from both models. This process is similar to those proposed by $[21,22]$ and it improved estimations in simulated examples (the particle system corresponding to $\eta_{2}=0.0216$ ) with measurements generated with the FMM. With this modification, used in both fixed and free form schemes, prior information has a major influence on results and loss of precision in estimations is compensated with an improvement in accuracy of the mean values. The results for simulated examples are not displayed since the emphasis in this work is related to the analysis of experimental examples.

Four experimental samples were studied. The same criterion explained for concentrated systems was used as well. In tables 1 to 4, estimations of mean radius and standard deviation of retrieved PSDs for each sample are shown with their respective confidence intervals and MAP solutions for Bayesian schemes. Previous estimations from a least-squares based (Levenberg-Marquardt) method reported in [5] are also included. Results from applying the Bayesian methodology are much more consistent with SEM estimations. This is particularly outstanding in recovered mean radii where differences between the LMA and the FMM derive in overestimated values when a least-squares approach with no prior information is used. Variances estimated following the Bayesian approach are reasonably wider than those obtained by SEM as it can be observed in MAP values. This can be explained by taking into account that this technique usually produce underestimates of distributions widths. It can be seen in fig. 1 that MAP estimated distributions are consistent with the histograms built from SEM micrographs where both Bayesian schemes give similar results. Greater differences correspond to most noisy measurements (samples 50PIB5 and 30PIB5). The data fittings of both proposed schemes and previous results are shown in fig.2.

As main conclusions of this work, a Bayesian methodology was successfully applied to combine data from two different experimental techniques. This methodology is specially recommended when reliable information is available and approximated models are used. 
Table 1. Estimations for sample 50PIB25

\begin{tabular}{cccc}
\hline Method & & $\bar{R} \pm \sigma_{\bar{R}}$ & $\sigma \pm \sigma_{\sigma}$ \\
\hline SEM Estimations & & $0.23 \pm 0.026$ & $0.12 \pm 0.0496$ \\
Least-squares (Levenberg- & & $0.321 \pm 0.011$ & $0.132 \pm 0.002$ \\
Marquardt) Approach & & & \\
Fixed-Form Bayesian & Statistics & $0.2318 \pm 0.0135$ & $0.1210 \pm 0.0075$ \\
Approach & MAP & 0.2456 & 0.1265 \\
Free-Form IBM Approach & Statistics & $0.2334 \pm 0.0092$ & $0.1213 \pm 0.0049$ \\
& MAP & 0.2332 & 0.1213 \\
\hline
\end{tabular}

Table 2. Estimations for sample 50PIB25_replica

\begin{tabular}{cccc}
\hline Method & & $\bar{R} \pm \sigma_{\bar{R}}$ & $\sigma \pm \sigma_{\sigma}$ \\
\hline SEM Estimations & & $0.19 \pm 0.0217$ & $0.091 \pm 0.0376$ \\
Least-squares (Levenberg- & & $0.333 \pm 0.008$ & $0.122 \pm 001$ \\
Marquardt) Approach & & & \\
Fixed-Form Bayesian & Statistics & $0.1915 \pm 0.0173$ & $0.1055 \pm 0.0083$ \\
Approach & MAP & 0.2092 & 0.1116 \\
Free-Form IBM Approach & Statistics & $0.1966 \pm 0.0061$ & $0.1147 \pm 0.0034$ \\
& MAP & 0.1955 & 0.1147 \\
\hline
\end{tabular}

Table 3. Estimations for sample 50PIB5

\begin{tabular}{cccc}
\hline Method & & $\bar{R} \pm \sigma_{\bar{R}}$ & $\sigma \pm \sigma_{\sigma}$ \\
\hline SEM Estimations & & $0.31 \pm 0.0343$ & $0.11 \pm .0426$ \\
Least-squares (Levenberg- & & $0.409 \pm 0.006$ & $0.0941 \pm 0.0033$ \\
Marquardt) Approach & & & \\
Fixed-Form Bayesian & Statistics & $0.3102 \pm 0.0158$ & $0.1073 \pm 0.0059$ \\
Approach & MAP & 0.3267 & 0.1109 \\
Free-Form IBM Approach & Statistics & $0.3274 \pm 0.0051$ & $0.1247 \pm 0.0065$ \\
& MAP & 0.3275 & 0.1246 \\
\hline
\end{tabular}

Table 4. Estimations for sample 30PIB5

\begin{tabular}{cccc}
\hline Method & & $\bar{R} \pm \sigma_{\bar{R}}$ & $\sigma \pm \sigma_{\sigma}$ \\
\hline SEM Estimations & & $0.21 \pm 0.0297$ & $0.054 \pm 0.0236$ \\
Least-squares (Levenberg- & & $0.282 \pm 0.011$ & $0.0952 \pm 0.0020$ \\
Marquardt) Approach & & & \\
Fixed-Form Bayesian & Statistics & $0.2207 \pm 0.0132$ & $0.0881 \pm 0.0337$ \\
Approach & MAP & 0.2352 & 0.0933 \\
Free-Form IBM Approach & Statistics & $0.2291 \pm 0.0031$ & $0.0872 \pm 0.018$ \\
& MAP & 0.2291 & 0.0872 \\
\hline
\end{tabular}



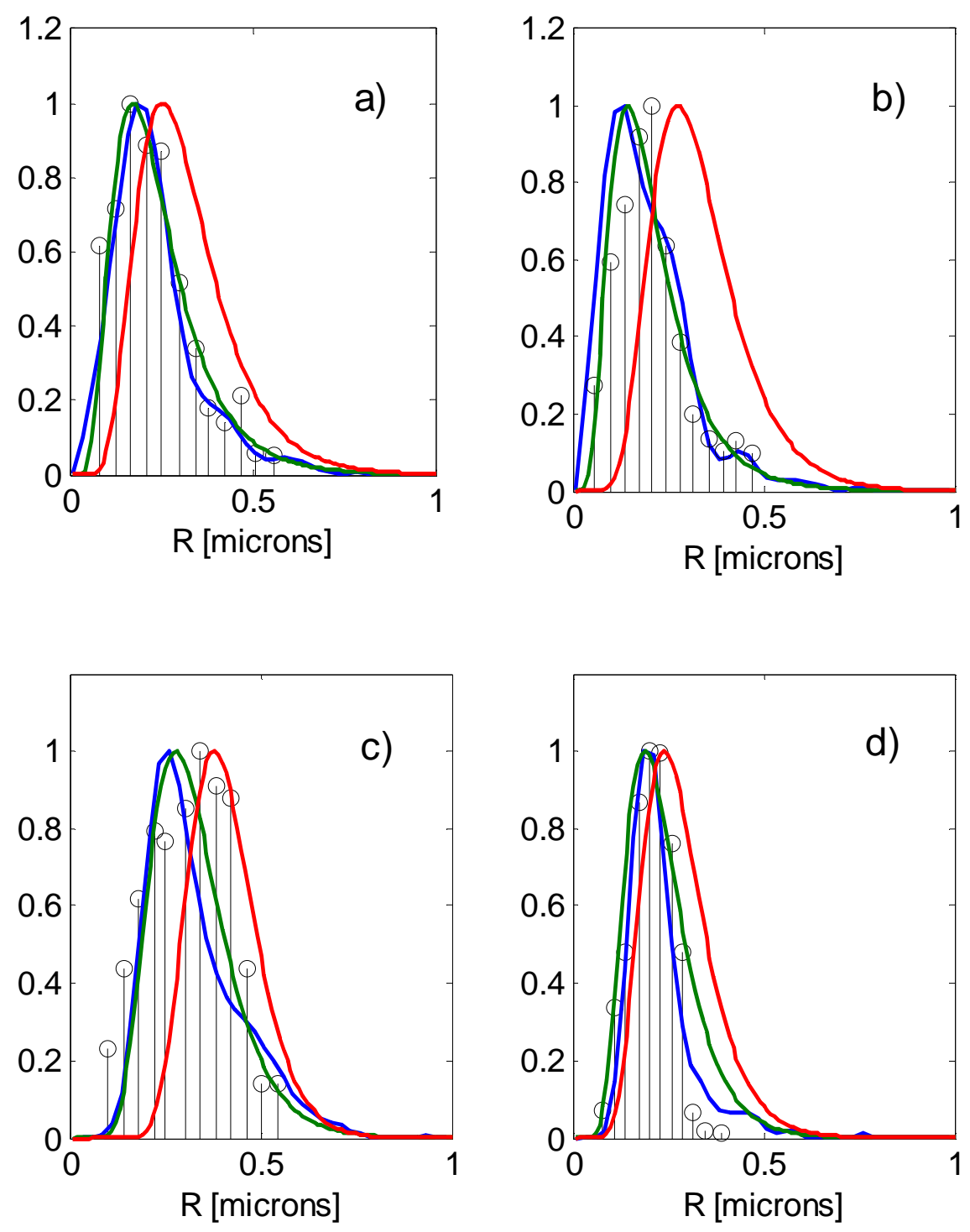

Figure 1. Estimated PSDs obtained with SEM (black bars) and by analysis of SLS data: using a least-squares method (red lines), a fixed-form Bayesian approach (green lines) and a freeform Iterative Bayesian Method (blue lines) for samples a) 50PIB25, b) 50PIB25_replica, c) 50 PIB5 and d)30PIB5 . 

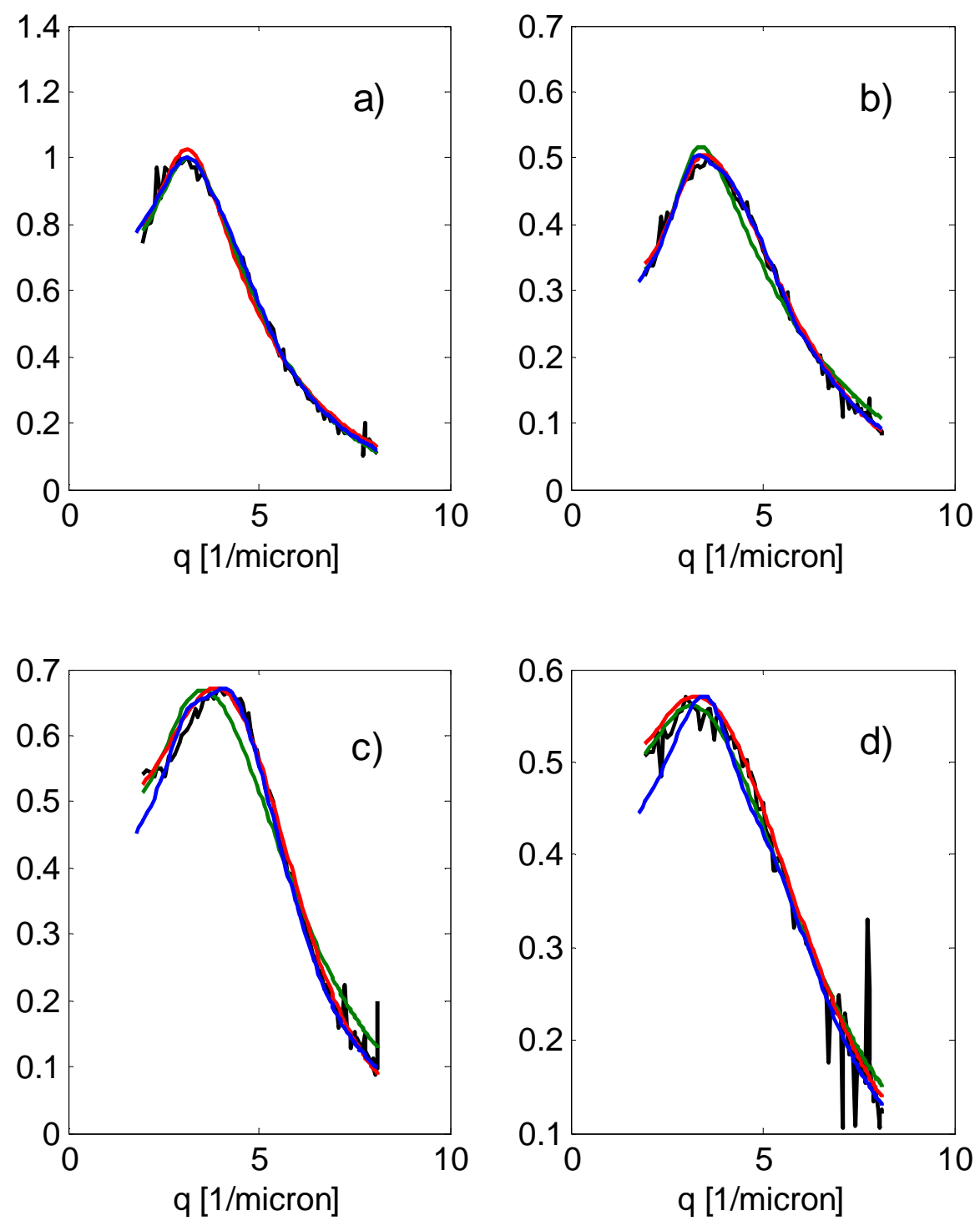

Figure 2. SLS Data fitting corresponding to the Least-squares approach (red), and MAP solutions from Fixed-form Bayesian approach (green) and Free-form Iterative Bayesian Method (blue) for samples a) 50PIB25, b) 50PIB25_replica, c) 50PIB5 and d)30PIB5. 


\section{Acknowledgements}

The authors acknowledge the financial support of the following institutions of Argentina: National University of Mar del Plata (UNMdP), National Research Council (CONICET) and National Agency for the Promotion of Science and Technology (ANPCyT)

\section{REFERENCES}

[1] Glatter O., Sieberer J., Schnablegger H., "A Comparative Study on Different Scattering Techniques and data evaluation methods for sizing of colloidal systems using light scattering", Part. Part. Syst. Charact. 8, 274-281, 1991.

[2] Pike E. R., Sabatier C., "Scattering and Inverse Scattering in Pure and Applied Science", Academic Press, London, 2002.

[3] Glatter O., Hofer M., "Interpretation of Elastic Light Scattering Data. III. Determination of Size Distributions of Polydisperse Systems", J. of Colloid and Interface Science 122, 496-506, 1988.

[4] Frontini, G. L., Fernández Berdaguer, E., "Inversion of Elastic Light Scattering measurements to determine Refractive Index and Particle Size Distribution of Polymeric Emulsions", Inverse Problems in Eng., 11, 329, 2003

[5] Otero F. A., Frontini G., Eliçabe G., "Evaluation of Light Scattering Models to Characterize Concentrated Polymer Particles embedded in a solid polymer matrix", J. of Polymer Science Part B: Polymer Physics 48 (9), 958-963, 2010.

[6] Vrij A., "Light Scattering of a Concentrated Multicomponent System of. Hard Spheres in the Percus-Yevick Approximation", J. Chem. Phys. 69, 1742, 1978.

[7] Vrij, A., "Mixtures of hard spheres in the Percus-Yevick approximation. Light scattering at finite angles", J. Chem. Phys. 71, 3267, 1979.

[8] Eliçabe G. E., Otero F., "Static Light Scattering of Concentrated Particle Systems in the Rayleigh-Debye-Gans Regime: Modelling and Data Analysis.", Particulate Science and Technology 28 (5), 485-497, 2010.

[9] Pedersen, J. S., Small-angle scattering from precipitates: Analysis by use of a polydisperse hard-sphere model, Phys. Rev. B 47, 657-665, 1993.

[10] Robert C. P., Castella G., "Monte Carlo Statistical Methods", Springer-Verlag, New York, 2004. 
[11] Geman, S.; Geman, D., "Stochastic Relaxation, Gibbs Distributions, and the Bayesian Restoration of Images", IEEE Transactions on Pattern Analysis and Machine Intelligence 6 (6), 721-741, 1984.

[12] Metropolis, N.; Rosenbluth, A.W.; Rosenbluth, M. N.; Teller, A. H.; Teller, E., "Equations of State Calculations by Fast Computing Machines", Journal of Chemical Physics 21 (6), 1087-1092, 1953.

[13] Hastings, W. K., "Monte Carlo Sampling Methods Using Markov Chains and Their Applications”, Biometrika 57 (1), 97-109, 1970.

[14] Chib S., Greenberg E., "Understanding the Metropolis-Hastings Algorithm", The American Statiscian 49, 327-335, 1995.

[15] Zanotto E.D., "Theoretical assessment of systematic errors in volume fraction determinations by microscopy methods", J. Materials Research 13, 2045, 1998.

[16] Geyer, C. J., Thompson E.A., "Annealing Markov chain Monte Carlo with applications to ancestral inference", J. Amer. Statist. Assoc. 90, 909-920, 1995.

[17] Gelman A., Gilks W. R., Roberts G. O., "Weak convergence and optimal scaling of random walk Metropolis algorithms", Ann. Appl. Prob. 7, 110-120, 1997.

[18] Wang, H., Li R., Tsai C.-L., "Tuning Parameter Selectors for the Smoothly Clipped Absolute Deviation Method", Biometrika 94, 553-568, 2007.

[19] Soulé, E. R., Elicabe G. E., Borrajo J., Williams R. J. J., “Analysis of the phase separation induced by a free-radical polymerization in solutions of polyisobutylene in isobornyl methacrylate", Ind. Eng. Chem. Res. 46, 7535-7542, 2007.

[20] Soulé, E. R., Elicabe G. E., "Determination of size distributions of concentrated polymer particles embedded in a solid polymer matrix", Part. Part. Syst. Charact. 25, 84-91, 2008.

[21] Currin, C., Mitchell, T., Morris, M., Ylvisaker, D., "Bayesian prediction of deterministic functions, with applications to the design and analysis of computer experiments", $J$. Am. Statist. Ass. 86, 953-963, 1992.

[22] Oakley, J., O'Hagan A., "Bayesian inference for the uncertainty distribution of computer model outputs", Biometrika 89, 769-784, 2002. 